\title{
Simultaneous Approximation for Generalized Srivastava-Gupta Operators
}

\author{
Tuncer Acar, ${ }^{1}$ Lakshmi Narayan Mishra, ${ }^{2,3}$ and Vishnu Narayan Mishra ${ }^{4}$ \\ ${ }^{1}$ Department of Mathematics, Faculty of Science and Arts, Kirikkale University, Yahsihan, 71450 Kirikkale, Turkey \\ ${ }^{2}$ Department of Mathematics, National Institute of Technology, Cachar District, Silchar, Assam 788 010, India \\ ${ }^{3}$ L. 1627 Awadh Puri Colony Beniganj, Phase III, Opposite to Industrial Training Institute (I.T.I.), Ayodhya Main Road, \\ Faizabad, Uttar Pradesh 224 001, India \\ ${ }^{4}$ Applied Mathematics and Humanities Department, Sardar Vallabhbhai National Institute of Technology, \\ Ichchhanath Mahadev Dumas Road, Surat District, Surat, Gujarat 395007, India
}

Correspondence should be addressed to Lakshmi Narayan Mishra; lakshminarayanmishra04@gmail.com

Received 19 May 2014; Accepted 9 October 2014

Academic Editor: Józef Banaś

Copyright (C) 2015 Tuncer Acar et al. This is an open access article distributed under the Creative Commons Attribution License, which permits unrestricted use, distribution, and reproduction in any medium, provided the original work is properly cited.

We introduce a new Stancu type generalization of Srivastava-Gupta operators to approximate integrable functions on the interval $(0, \infty)$ and estimate the rate of convergence for functions having derivatives of bounded variation. Also we present simultenaous approximation by new operators in the end of the paper.

\section{Introduction}

To approximate integrable functions on the interval $(0, \infty)$, Srivastava and Gupta [1] introduced a general sequence of linear positive operators $G_{n, c}$ as follows:

$$
\begin{aligned}
G_{n, c}(f ; x)= & n \sum_{k=1}^{\infty} p_{n, k}(x ; c) \int_{0}^{\infty} p_{n+c, k-1}(t ; c) f(t) d t \\
& +p_{n, 0}(x ; c) f(0)
\end{aligned}
$$

for a function $f \in H_{\alpha}(0, \infty)$, where $H_{\alpha}(0, \infty)(\alpha \geq 0)$ is the class of locally integrable functions defined on $(0, \infty)$ and satisfying the growth condition

$$
\begin{gathered}
|f(t)| \leq M t^{\alpha} \quad(M>0 ; \alpha \geq 0 ; t \rightarrow \infty), \\
p_{n, k}(x ; c)=\frac{(-x)^{k}}{k !} \phi_{n, c}^{(k)}(x), \\
\phi_{n, c}(x)= \begin{cases}e^{-n x}, & c=0 \\
(1+c x)^{-n / c}, & c \in \mathbb{N}:=\{1,2,3, \ldots\} .\end{cases}
\end{gathered}
$$

The general sequence of operators $G_{n, c}$ has many interesting properties in approximation theory, which is an interesting area of research in the present era, and several researchers have studied these operators; we can mention some important studies on these operators (see [1-3]). In [4], author introduced King and Stancu type generalization of Srivastava-Gupta operators and presented some direct results. Also, Verma and Agrawal [5] introduced a new generalization of Srivastava-Gupta operators and studied the rate of convergence for the functions having the derivatives of bounded variation (BV). The rate of convergence for the functions having the derivatives of (BV) is an active area of research and many researchers studied this direction. We refer the readers to [6-10] and references therein.

Stancu $[11,12]$ introduced generalizations of Bernstein polynomials with one and two parameters (resp.), satisfying the condition $0 \leq \alpha \leq \beta$, as

$$
\begin{array}{r}
s_{n}^{\alpha}(f, x)=\sum_{k=0}^{n} f\left(\frac{k}{n}\right)\left(\begin{array}{l}
n \\
k
\end{array}\right) \frac{\prod_{s=0}^{k-1}(x+\alpha s) \prod_{s=0}^{n-k-1}(1-x+\alpha s)}{\prod_{s=0}^{n-1}(1+\alpha s)} \\
0 \leq x \leq 1,
\end{array}
$$




$$
\begin{array}{r}
s_{n}^{\alpha, \beta}(f, x)=\sum_{k=0}^{n} f\left(\frac{k+\alpha}{n+\beta}\right)\left(\begin{array}{l}
n \\
k
\end{array}\right) x^{k}(1-x)^{n-k} \\
0 \leq x \leq 1,
\end{array}
$$

for any $f \in C[0,1]$. Stancu type generalization of approximation operators present better approach depending on $\alpha, \beta$. Therefore, this kind of generalizations and their approximation properties have been studied intensively. We refer the readers to [13-17] and references therein. Mishra et al. [18, 19], V. N. Mishra, and L. N. Mishra [20] have established very interesting results on approximation properties of various functional classes using different types of positive linear summability operators.

The purpose of this paper is to introduce a new Stancu type generalization of the operators defined in [5] as

$$
\begin{aligned}
G_{n, r, c}^{(\alpha, \beta)}(f ; x)= & \frac{n \Gamma((n / c)+r) \Gamma((n / c)-r+1)}{\Gamma((n / c)+1) \Gamma(n / c)} \sum_{k=0}^{\infty} p_{n+r c, k}(x ; c) \\
& \times \int_{0}^{\infty} p_{n-(r-1) c, k+r-1}(t ; c) f\left(\frac{n t+\alpha}{n+\beta}\right) d t .
\end{aligned}
$$

By the definition of operators, it is clear that $G_{n, r, c}^{(\alpha, \beta)}(f ; x)$ is positive and linear. For $\alpha=\beta=0, G_{n, r, c}^{(0,0)}(f ; x)$ reduces to operators defined in [5]. In this study we obtain the rate of convergence for the functions having the derivatives of bounded variation. Also, in the end of the paper, we study the simultaneous approximation.

\section{Auxiliary Results}

In order to prove our main results, we need the following lemmas.

Lemma 1. Let the mth order moment be defined as

$$
\begin{aligned}
U_{n, r, m}^{\alpha, \beta}(x)= & G_{n, c}^{(\alpha, \beta)}\left((t-x)^{m} ; x\right) \\
= & (n-r c) \sum_{k=0}^{\infty} p_{n+r c, k}(x ; c) \\
& \times \int_{0}^{\infty} p_{n-(r-1) c, k+r-1}(t ; c)\left(\frac{n t+\alpha}{n+\beta}-x\right)^{m} d t,
\end{aligned}
$$

where $n, m \in \mathbb{N} \cup\{0\}$, and then, for $n>(m+r+1) c$, we have the following recurrence relation:

$$
\begin{aligned}
(n- & (r+m+1) c)(n+\beta) U_{n, r, m+1}(x) \\
= & n x(1+c x)\left[\left(U_{n, r, m}^{\alpha, \beta}(x)\right)^{\prime}+m U_{n, r, m-1}^{\alpha, \beta}(x)\right] \\
+ & U_{n, r, m}^{\alpha, \beta}(x) \\
& \times[(m+r+(n+r c) x) n+(\alpha-(n+\beta) x) \\
& \quad \times(n-(r+2 m+1) c)] \\
+ & U_{n, r, m-1}^{\alpha, \beta}(x) \\
& \times\left[\frac{c m(\alpha-(n+\beta) x)^{2}-m n(\alpha-(n+\beta) x)}{n+\beta}\right],
\end{aligned}
$$

$$
\begin{aligned}
U_{n, r, 0}^{\alpha, \beta}(x)= & 1, \\
U_{n, r, 1}^{\alpha, \beta}(x)= & \frac{\alpha-(n+\beta) x}{n+\beta}+\frac{n(r+(n+r c) x)}{(n-(r+1) c)(n+\beta)} \\
U_{n, r, 2}^{\alpha, \beta}(x)= & \frac{n x(1+c x)}{(n-(r+1) c)(n-(r+2) c)(n+\beta)^{2}} \\
& +\left(\frac{\alpha}{n+\beta}-x+\frac{n(r+(n+r c) x)}{(n-(r+1) c)(n+\beta)}\right) \\
& \times \frac{n(1+r+(n+r c) x)}{(n-(r+2) c)(n+\beta)} \\
& +\left(\frac{\alpha}{n+\beta}-x+\frac{n(r+(n+r c) x)}{(n-(r+1) c)(n+\beta)^{2}}\right) \\
& \times(\alpha-(n+\beta) x) \\
& +\frac{(\alpha-(n+\beta) x)(c(\alpha-(n+\beta) x)-n)}{(n-(r+2) c)(n+\beta)^{2}}
\end{aligned}
$$

Furthermore, $U_{n, r, m}^{\alpha, \beta}(x)$ is polynomial of degree $m$ in $x$ and

$$
U_{n, r, m}^{\alpha, \beta}(x)=O\left((n+\beta)^{-[(m+1) / 2]}\right) .
$$

Proof. By definition of $U_{n, r, m}^{\alpha, \beta}(x)$, taking the derivative of $U_{n, r, m}^{\alpha, \beta}(x)$, we get

$$
\begin{aligned}
\left(U_{n, r, m}^{\alpha, \beta}(x)\right)^{\prime} & \\
= & -(n-r c) m \sum_{k=0}^{\infty} p_{n+r c, k}(x ; c) \\
& \times \int_{0}^{\infty} p_{n-(r-1) c, k+r-1}(t ; c)\left(\frac{n t+\alpha}{n+\beta}-x\right)^{m-1} d t \\
& +(n-r c) \sum_{k=0}^{\infty} p_{n+r c, k}^{\prime}(x ; c) \\
& \times \int_{0}^{\infty} p_{n-(r-1) c, k+r-1}(t ; c)\left(\frac{n t+\alpha}{n+\beta}-x\right)^{m} d t \\
= & -m U_{n, r, m-1}^{\alpha, \beta}(x)+(n-r c) \sum_{k=0}^{\infty} p_{n+r c, k}^{\prime}(x ; c) \\
& \times \int_{0}^{\infty} p_{n-(r-1) c, k+r-1}(t ; c)\left(\frac{n t+\alpha}{n+\beta}-x\right)^{m} d t .
\end{aligned}
$$

Hence, using the identity

$$
x(1+c x) p_{n+r c, k}^{\prime}(x ; c)=(k-(n+r c) x) p_{n+r c, k}(x ; c)
$$


we have

$$
\begin{aligned}
x(1+ & c x)\left[\left(U_{n, r, m}^{\alpha, \beta}(x)\right)^{\prime}+m U_{n, r, m-1}^{\alpha, \beta}(x)\right] \\
= & (n-r c) \sum_{k=0}^{\infty}(k-(n+r c) x) p_{n+r c, k}(x ; c) \\
& \times \int_{0}^{\infty} p_{n-(r-1) c, k+r-1}(t ; c)\left(\frac{n t+\alpha}{n+\beta}-x\right)^{m} d t \\
= & (n-r c) \sum_{k=0}^{\infty} k p_{n+r c, k}(x ; c) \\
& \times \int_{0}^{\infty} p_{n-(r-1) c, k+r-1}(t ; c)\left(\frac{n t+\alpha}{n+\beta}-x\right)^{m} d t \\
& -(n+r c) x U_{n, r, m}^{\alpha, \beta}(x) \\
= & I-(n+r c) x U_{n, r, m}^{\alpha, \beta}(x) .
\end{aligned}
$$

We can write $I$ as

$$
\begin{aligned}
I=[ & (n-r c) \sum_{k=0}^{\infty} p_{n+r c, k}(x ; c) \\
& \times \int_{0}^{\infty}[k+r-1-(n-(r-1) c) t] p_{n-(r-1) c, k+r-1} \\
& \quad \times(t ; c)\left(\frac{n t+\alpha}{n+\beta}-x\right)^{m} d t \\
& +(n-r c)(n-(r-1) c) \sum_{k=0}^{\infty} p_{n+r c, k}(x ; c) \\
& \times \int_{0}^{\infty} p_{n-(r-1) c, k+r-1}(t ; c) t\left(\frac{n t+\alpha}{n+\beta}-x\right)^{m} d t \\
& -(r-1)(n-r c) \sum_{k=0}^{\infty} p_{n+r c, k}(x ; c) \\
& \left.\times \int_{0}^{\infty} p_{n-(r-1) c, k+r-1}(t ; c)\left(\frac{n t+\alpha}{n+\beta}-x\right)^{m} d t\right] \\
= & I_{1}+I_{2}-(r-1) U_{n, r, m}^{\alpha, \beta}(x) .
\end{aligned}
$$

To estimate $I_{2}$ using $t=((n+\beta) / n)[(((n t+\alpha) /(n+\beta))-x)-$ $((\alpha /(n+\beta))-x)]$, we have

$$
\begin{aligned}
I_{2}=\frac{(n-(r-1) c)(n+\beta)}{n} \\
\times\left[(n-r c) \sum_{k=0}^{\infty} p_{n+r c, k}(x ; c)\right. \\
\quad \times \int_{0}^{\infty} p_{n-(r-1) c, k+r-1}(t ; c)\left(\frac{n t+\alpha}{n+\beta}-x\right)^{m+1} d t \\
\quad-\left(\frac{\alpha}{n+\beta}-x\right)(n-r c) \sum_{k=0}^{\infty} p_{n+r c, k}(x ; c) \\
\left.\quad \times \int_{0}^{\infty} p_{n-(r-1) c, k+r-1}(t ; c)\left(\frac{n t+\alpha}{n+\beta}-x\right)^{m} d t\right],
\end{aligned}
$$

$$
\begin{aligned}
I_{2}= & \frac{(n-(r-1) c)(n+\beta)}{n} \\
\times & {\left[(n-r c) \sum_{k=0}^{\infty} p_{n+r c, k}(x ; c)\right.} \\
& \times \int_{0}^{\infty} p_{n-(r-1) c, k+r-1}(t ; c)\left(\frac{n t+\alpha}{n+\beta}-x\right)^{m+1} d t \\
& -\left(\frac{\alpha}{n+\beta}-x\right) \\
& \times\left((n-r c) \sum_{k=0}^{\infty} p_{n+r c, k}(x ; c)\right. \\
& \times \int_{0}^{\infty} p_{n-(r-1) c, k+r-1}(t ; c) \\
\times & \left.\left.\times\left(\frac{n t+\alpha}{n+\beta}-x\right)^{m} d t\right)\right] \\
= & \left(n-\frac{(r-1) c)(n+\beta)}{n}(x)-\left(\frac{\alpha}{n+\beta}-x\right) U_{n, r, m}^{\alpha, \beta}(x)\right] .
\end{aligned}
$$

Next to estimate $I_{1}$ using the equality

$$
\begin{aligned}
t(1+c t) p_{n-(r-1) c, k+r-1}^{\prime}(t ; c) \\
\quad=[(k+r-1)-(n-(r-1) c) t] p_{n-(r-1) c, k+r-1}(t ; c),
\end{aligned}
$$

we have

$$
\begin{aligned}
I_{1}= & (n-r c) \sum_{k=0}^{\infty} p_{n+r c, k}(x ; c) \\
& \times \int_{0}^{\infty} p_{n-(r-1) c, k+r-1}^{\prime}(t ; c) t\left(\frac{n t+\alpha}{n+\beta}-x\right)^{m} d t \\
& +c(n-r c) \sum_{k=0}^{\infty} p_{n+r c, k}(x ; c) \\
& \times \int_{0}^{\infty} p_{n-(r-1) c, k+r-1}^{\prime}(t ; c) t^{2}\left(\frac{n t+\alpha}{n+\beta}-x\right)^{m} d t \\
= & \mathscr{J}_{1}+\mathscr{J}_{2} .
\end{aligned}
$$


Putting $t=((n+\beta) / n)[(((n t+\alpha) /(n+\beta))-x)-((\alpha /(n+\beta))-x)]$, we get

$$
\begin{aligned}
\mathscr{J}_{1}=\frac{n+\beta}{n} & \\
\times & {\left[(n-r c) \sum_{k=0}^{\infty} p_{n+r c, k}(x ; c)\right.} \\
& \times \int_{0}^{\infty} p_{n-(r-1) c, k+r-1}^{\prime}(t ; c)\left(\frac{n t+\alpha}{n+\beta}-x\right)^{m+1} d t \\
& -\left(\frac{\alpha}{n+\beta}-x\right)(n-r c) \sum_{k=0}^{\infty} p_{n+r c, k}(x ; c) \\
& \left.\times \int_{0}^{\infty} p_{n-(r-1) c, k+r-1}^{\prime}(t ; c)\left(\frac{n t+\alpha}{n+\beta}-x\right)^{m} d t\right] .
\end{aligned}
$$

Now integrating by parts, we get

$$
\begin{aligned}
\mathscr{J}_{1}= & -(m+1)(n-r c) \sum_{k=0}^{\infty} p_{n+r c, k}(x ; c) \\
& \times \int_{0}^{\infty} p_{n-(r-1) c, k+r-1}(t ; c)\left(\frac{n t+\alpha}{n+\beta}-x\right)^{m} d t \\
& +m\left(\frac{\alpha}{n+\beta}-x\right)(n-r c) \sum_{k=0}^{\infty} p_{n+r c, k}(x ; c) \\
& \times \int_{0}^{\infty} p_{n-(r-1) c, k+r-1}(t ; c)\left(\frac{n t+\alpha}{n+\beta}-x\right)^{m-1} d t \\
= & -(m+1) \\
& \times\left[(n-r c) \sum_{k=0}^{\infty} p_{n+r c, k}(x ; c)\right. \\
& \left.\times \int_{0}^{\infty} p_{n-(r-1) c, k+r-1}(t ; c)\left(\frac{n t+\alpha}{n+\beta}-x\right)^{m} d t\right] \\
& +m\left(\frac{\alpha}{n+\beta}-x\right) \\
& \times\left[(n-r c) \sum_{k=0}^{\infty} p_{n+r c, k}(x ; c)\right. \\
& \left.\times \int_{0}^{\infty} p_{n-(r-1) c, k+r-1}(t ; c)\left(\frac{n t+\alpha}{n+\beta}-x\right)^{m-1} d t\right] \\
& {[m+1) U_{n, r, m}^{\alpha, \beta}(x) } \\
& {\left[(x) U_{n, r, m-1}^{\alpha, \beta}(x) .\right.} \\
& {[(x)}
\end{aligned}
$$

Proceeding in a similar manner, we obtain the estimate $\mathscr{J}_{2}$ as

$$
\begin{aligned}
\mathscr{J}_{2}= & -\frac{c(m+2)(n+\beta)}{n} U_{n, r, m+1}(x) \\
& +\frac{2 c(m+1)(n+\beta)}{n}\left(\frac{\alpha}{n+\beta}-x\right) U_{n, r, m}(x) \\
& -\frac{c m(n+\beta)}{n}\left(\frac{\alpha}{n+\beta}-x\right)^{2} U_{n, r, m-1}^{\alpha, \beta}(x) .
\end{aligned}
$$

Combining the equations, we have

$$
\begin{aligned}
(n-(r+ & m+1) c)(n+\beta) U_{n, r, m+1}^{\alpha, \beta}(x) \\
= & n x(1+c x)\left[\left(U_{n, r, m}^{\alpha, \beta}(x)\right)^{\prime}+m U_{n, r, m-1}^{\alpha, \beta}(x)\right] \\
& +U_{n, r, m}^{\alpha, \beta}(x) \\
& \times[(m+r+(n+r c) x) n+(\alpha-(n+\beta) x) \\
& \times(n-(r+2 m+1) c)]+U_{n, r, m-1}^{\alpha, \beta}(x) \\
& \times\left[\frac{c m(\alpha-(n+\beta) x)^{2}-m n(\alpha-(n+\beta) x)}{n+\beta}\right]
\end{aligned}
$$

which is the desired result.

Moments for $m=0,1,2$ can be easily obtained by using the above recurrence relation.

Remark 2. For sufficiently large $n, C>2$, and $x \in(0, \infty)$, it can be seen from Lemma 1 that

$$
U_{n, r, 2}^{\alpha, \beta}(x) \leq \frac{C \sigma_{r, c}^{\alpha, \beta}(x)}{n+\beta}
$$

where $\sigma_{r, c}^{\alpha, \beta}(x)=[x(1+c x)+x(\alpha+\beta x+r(1+c x))]$ for the convenient notation.

Remark 3. By using Cauchy-Schwarz inequality, it follows from Remark 2 that, for sufficiently large $n, C>2$, and $x \in(0, \infty)$,

$$
\begin{aligned}
& (n-r c) \sum_{k=0}^{\infty} p_{n+r c, k}(x ; c) \\
& \quad \times \int_{0}^{\infty} p_{n-(r-1) c, k+r-1}(t ; c)\left|\frac{n t+\alpha}{n+\beta}-x\right| d t \\
& \leq\left[U_{n, r, 2}^{\alpha, \beta}(x)\right]^{1 / 2} \leq \sqrt{\frac{C \sigma_{r, c}^{\alpha, \beta}(x)}{n+\beta}} .
\end{aligned}
$$


Lemma 4. Let $x \in(0, \infty)$ and $C>2$; then, for sufficiently large $n$, we have

$$
\begin{aligned}
\lambda_{n, r}(x, y)= & (n-r c) \sum_{k=0}^{\infty} p_{n+r c, k}(x ; c) \\
& \times \int_{0}^{y} p_{n-(r-1) c, k+r-1}(t ; c) d t \\
\leq & \frac{C x(1+c x)}{n(x-y)^{2}}, \quad 0 \leq y \leq x, \\
1-\lambda_{n, r}(x, z)= & (n-r c) \sum_{k=0}^{\infty} p_{n+r c, k}(x ; c) \\
& \times \int_{z}^{\infty} p_{n-(r-1) c, k+r-1}(t ; c) d t \\
\leq & \frac{C x(1+c x)}{n(z-x)^{2}}, \quad x \leq z \leq \infty .
\end{aligned}
$$

Proof. We give the proof for only first inequality, and the other is similar. Using Remark 2 with $\alpha=\beta=0$, for sufficiently large $n$ and $0 \leq y \leq x$ and $((n t+\alpha) /(n+\beta)) \leq t$, we have

$$
\begin{aligned}
\lambda_{n, r}(x, y)= & (n-r c) \sum_{k=0}^{\infty} p_{n+r c, k}(x ; c) \\
& \times \int_{0}^{y} p_{n-(r-1) c, k+r-1}(t ; c) d t \\
\leq & (n-r c) \sum_{k=0}^{\infty} p_{n+r c, k}(x ; c) \\
& \times \int_{0}^{y} p_{n-(r-1) c, k+r-1}(t ; c) \frac{(t-x)^{2}}{(y-x)^{2}} d t \\
\leq & \frac{C x(1+c x)}{n(x-y)^{2}} .
\end{aligned}
$$

Lemma 5. Suppose $f$ is $s$ times differentiable on $[0, \infty)$ such that $f^{(s-1)}(t)=O\left(t^{\alpha}\right)$, for some integer $\alpha>0$ as $t \rightarrow \infty$. Then, for any $r, s \in \mathbb{N}_{0}$, and $n>\max \{\alpha, r+s\}$, we have

$$
D^{s} G_{n, r, c}^{(\alpha, \beta)}(f ; x)=\left(\frac{n}{n+\beta}\right)^{s} G_{n, r+s, c}^{(\alpha, \beta)}(f ; x)\left(D^{s} f, x\right) .
$$

Proof. Using the identity

$$
p_{n, k}^{\prime}(x)=n\left[p_{n+c, k-1}(x, c)-p_{n+c, k}(x, c)\right] .
$$

One can observe that, even in case $k=0$, the above identity is true with the condition $p_{n+c \text {, negative }}(x, c)=0$. Thus, applying (26), we have

$$
\begin{aligned}
& D\left[G_{n, r, c}^{(\alpha, \beta)}\right](f ; x) \\
& =\frac{n \Gamma((n / c)+r) \Gamma((n / c)-r+1)}{\Gamma((n / c)+1) \Gamma(n / c)} \sum_{k=0}^{\infty} D p_{n+r c, k}(x ; c) \\
& \times \int_{0}^{\infty} p_{n-(r-1) c, k+r-1}(t ; c) f\left(\frac{n t+\alpha}{n+\beta}\right) d t \\
& =\frac{n \Gamma((n / c)+r) \Gamma((n / c)-r+1)}{\Gamma((n / c)+1) \Gamma(n / c)} \\
& \times \sum_{k=0}^{\infty}(n+r c)\left[p_{n+(r+1) c, k-1}(x, c)-p_{n+(r+1) c, k}(x, c)\right] \\
& \times \int_{0}^{\infty} p_{n-(r-1) c, k+r-1}(t ; c) f\left(\frac{n t+\alpha}{n+\beta}\right) d t \\
& =\frac{n(n+r c) \Gamma((n / c)+r) \Gamma((n / c)-r+1)}{\Gamma((n / c)+1) \Gamma(n / c)} \\
& \times \sum_{k=0}^{\infty} p_{n+(r+1) c, k}(x, c) \\
& \times \int_{0}^{\infty}\left[p_{n-(r-1) c, k+r}(t ; c)-p_{n-(r-1) c, k+r-1}(t ; c)\right] \\
& \times f\left(\frac{n t+\alpha}{n+\beta}\right) d t \\
& =\frac{-n(n+r c) \Gamma((n / c)+r) \Gamma((n / c)-r+1)}{(n-r c) \Gamma((n / c)+1) \Gamma(n / c)} \\
& \times \sum_{k=0}^{\infty} p_{n+(r+1) c, k}(x, c) \\
& \times \int_{0}^{\infty} D p_{n-r c, k+r}(t ; c) f\left(\frac{n t+\alpha}{n+\beta}\right) d t \\
& =\frac{n^{2} \Gamma((n / c)+r+1) \Gamma((n / c)-r)}{(n+\beta) \Gamma((n / c)+1) \Gamma(n / c)} \\
& \times \sum_{k=0}^{\infty} p_{n+(r+1) c, k}(x, c) \\
& \times \int_{0}^{\infty} p_{n-r c, k+r}(t ; c) D f\left(\frac{n t+\alpha}{n+\beta}\right) d t \\
& =\frac{n}{(n+\beta)}\left[G_{n, r+1, c}^{(\alpha, \beta)}\right](D f ; x),
\end{aligned}
$$


which means that the identity is satisfied for $s=1$. Let us suppose that the result holds for $s=m$; that is,

$$
\begin{aligned}
& D^{m} G_{n, r, c}^{(\alpha, \beta)}(f ; x) \\
&=\left(\frac{n}{n+\beta}\right)^{m} G_{n, r+m, c}^{(\alpha, \beta)}(f ; x)\left(D^{m} f, x\right) \\
&=\left(\frac{n}{n+\beta}\right)^{m} \\
& \times \frac{n \Gamma((n / c)+r+m) \Gamma((n / c)-r-m+1)}{\Gamma((n / c)+1) \Gamma(n / c)} \\
& \times \sum_{k=0}^{\infty} p_{n+(r+m) c, k}(x ; c) \\
& \times \int_{0}^{\infty} p_{n-(r+m-1) c, k+r+m-1}(t ; c) D^{m} f\left(\frac{n t+\alpha}{n+\beta}\right) d t .
\end{aligned}
$$

Also, from (26) we can write

$$
\begin{aligned}
& D^{m+1} G_{n, r, c}^{(\alpha, \beta)}(f ; x) \\
& =\left(\frac{n}{n+\beta}\right)^{m} \\
& \times \frac{n \Gamma((n / c)+r+m) \Gamma((n / c)-r-m+1)}{\Gamma((n / c)+1) \Gamma(n / c)} \\
& \times \sum_{k=0}^{\infty} D p_{n+(r+m) c, k}(x ; c) \\
& \times \int_{0}^{\infty} p_{n-(r+m-1) c, k+r+m-1}(t ; c) D^{m} f\left(\frac{n t+\alpha}{n+\beta}\right) d t \\
& =\left(\frac{n}{n+\beta}\right)^{m} \\
& \times \frac{n \Gamma((n / c)+r+m) \Gamma((n / c)-r-m+1)}{\Gamma((n / c)+1) \Gamma(n / c)} \\
& \times \sum_{k=0}^{\infty}(n+(r+m) c) \\
& \times\left[p_{n+(r+m+1) c, k-1}(x, c)-p_{n+(r+m+1) c, k}(x, c)\right] \\
& \times \int_{0}^{\infty} p_{n-(r+m-1) c, k+r+m-1}(t ; c) D^{m} f\left(\frac{n t+\alpha}{n+\beta}\right) d t \\
& =\left(\frac{n}{n+\beta}\right)^{m} \\
& \times \frac{c n \Gamma((n / c)+r+m+1) \Gamma((n / c)-r-m+1)}{\Gamma((n / c)+1) \Gamma(n / c)} \\
& \times \sum_{k=0}^{\infty} p_{n+(r+m+1) c, k}(x ; c) \\
& \times \int_{0}^{\infty}\left[p_{n-(r+m-1) c, k+r+m}(t ; c)\right. \\
& \left.-p_{n-(r+m-1) c, k+r+m-1}(t ; c)\right] D^{m} f\left(\frac{n t+\alpha}{n+\beta}\right) d t
\end{aligned}
$$

$$
\begin{aligned}
= & -\left(\frac{n}{n+\beta}\right)^{m} \\
& \times \frac{c n \Gamma((n / c)+r+m+1) \Gamma((n / c)-r-m+1)}{\Gamma((n / c)+1) \Gamma(n / c)} \\
& \times \sum_{k=0}^{\infty} p_{n+(r+m+1) c, k}(x ; c) \\
& \times \int_{0}^{\infty} \frac{D p_{n-(r+m) c, k+r+m}(t ; c)}{n-(r+m-1) c} D^{m} f\left(\frac{n t+\alpha}{n+\beta}\right) d t
\end{aligned}
$$

and, integrating by parts the last integral, we have

$$
\begin{aligned}
D^{m+1} & G_{n, r, c}^{(\alpha, \beta)}(f ; x) \\
= & \left(\frac{n}{n+\beta}\right)^{m+1} \\
& \times \frac{n \Gamma((n / c)+r+m+1) \Gamma((n / c)-r-m)}{\Gamma((n / c)+1) \Gamma(n / c)} \\
& \times \sum_{k=0}^{\infty} p_{n+(r+m+1) c, k}(x ; c) \\
& \times \int_{0}^{\infty} p_{n-(r+m) c, k+r+m}(t ; c) D^{m+1} f\left(\frac{n t+\alpha}{n+\beta}\right) d t .
\end{aligned}
$$

Hence we have

$$
\begin{aligned}
D^{m+1} G_{n, r, c}^{(\alpha, \beta)}(f ; x)= & \left(\frac{n}{n+\beta}\right)^{m+1} \\
& \times G_{n, r+m+1, c}^{(\alpha, \beta)}(f ; x)\left(D^{m+1} f, x\right),
\end{aligned}
$$

in which the result is true for $s=m+1$, and hence by mathematical induction the proof of the lemma is completed.

\section{Main Results}

Throughout the paper by $D B_{q}(0, \infty)$ we denote the class of absolutely continuous functions $f$ on $(0, \infty)$ (where $q$ is a some positive integer) satisfying the conditions:

(i) $|f(t)| \leq C_{1} t^{q}$ and $C_{1}>0$,

(ii) the function $f$ has the first derivative on the interval $(0, \infty)$ which coincide almost everywhere with a function which is of bounded variation on every finite subinterval of $(0, \infty)$. It can be observed that for all functions $f \in D B_{q}(0, \infty)$ we can have the representation

$$
f(x)=f(c)+\int_{c}^{x} \psi(t) d t, \quad x \geq c \geq 0 .
$$


Theorem 6. Let $f \in D B_{q}(0, \infty), q>0$, and $x \in(0, \infty)$. Then, for $C>2$ and sufficiently large $n$, we have

$$
\begin{aligned}
& \left|\frac{(\Gamma(n / c))^{2}}{\Gamma((n / c)+r) \Gamma((n / c)-r)} G_{n, r, c}^{(\alpha, \beta)}(f ; x)-f(x)\right| \\
& \leq \frac{C(1+c x)}{n} \sum_{k=1}^{[\sqrt{n}]} \bigvee_{x-(x / k)}^{x+(x / k)}\left(f_{x}^{\prime}(x)\right)+\frac{x}{\sqrt{n}} \bigvee_{x-(x / \sqrt{n})}^{x+(x / \sqrt{n})}\left(f_{x}^{\prime}(x)\right) \\
& \quad+\frac{C(1+c x)}{n x}\left|f(2 x)-f(x)-x f^{\prime}\left(x^{+}\right)+\right| f(x)|| \\
& \quad+O\left(n^{-q}\right)+\left|f^{\prime}\left(x^{+}\right)\right| \sqrt{\frac{C x(1+c x)}{n}} \\
& \quad+\sqrt{\frac{C \sigma_{r, c}^{\alpha, \beta}(x)}{n+\beta}} \mid \frac{f^{\prime}\left(x^{+}\right)-f^{\prime}\left(x^{-}\right) \mid}{2}+\frac{\left|f^{\prime}\left(x^{+}\right)+f^{\prime}\left(x^{-}\right)\right|}{2} \\
& \quad \times\left(\frac{(\alpha-\beta x)(n-c(r+1))+2 n r c x+n x c+n r}{(n-(r+1) c)(n+\beta)}\right),
\end{aligned}
$$

where $C$ is a constant which may be different on each occurrence.

Proof. Using the mean value theorem, we have

$$
\begin{aligned}
& \frac{(\Gamma(n / c))^{2}}{\Gamma((n / c)+r) \Gamma((n / c)-r)} G_{n, r, c}^{(\alpha, \beta)}(f ; x)-f(x) \\
& =(n-r c) \sum_{k=0}^{\infty} p_{n+r c, k}(x ; c) \\
& \quad \times \int_{0}^{\infty} p_{n-(r-1) c, k+r-1}(t ; c)\left[f\left(\frac{n t+\alpha}{n+\beta}\right)-f(x)\right] d t \\
& =\int_{0}^{\infty}\left(\int_{x}^{(n t+\alpha) /(n+\beta)}(n-r c) \sum_{k=0}^{\infty} p_{n+r c, k}(x ; c)\right. \\
& \left.\quad \times p_{n-(r-1) c, k+r-1}(t ; c) f^{\prime}(u) d u\right) d t .
\end{aligned}
$$

Also, using the identity

$$
\begin{aligned}
f^{\prime}(u)= & \frac{f^{\prime}\left(x^{+}\right)+f^{\prime}\left(x^{-}\right)}{2}+\left(f^{\prime}\right)_{x}(u) \\
& +\frac{f^{\prime}\left(x^{+}\right)-f^{\prime}\left(x^{-}\right)}{2} \operatorname{sgn}(u-x) \\
& +\left[f^{\prime}(x)-\frac{f^{\prime}\left(x^{+}\right)+f^{\prime}\left(x^{-}\right)}{2}\right] \chi_{x}(u),
\end{aligned}
$$

where

$$
\chi_{x}(u)= \begin{cases}1, & u=x \\ 0, & u \neq x\end{cases}
$$

we have

$$
\begin{aligned}
&(n-r c) \sum_{k=0}^{\infty} p_{n+r c, k}(x ; c) \\
& \times \int_{0}^{\infty}\left(\int_{x}^{t}\left[f^{\prime}(x)-\frac{f^{\prime}\left(x^{+}\right)+f^{\prime}\left(x^{-}\right)}{2}\right] \chi_{x}(u) d u\right) \\
& \quad \times p_{n-(r-1) c, k+r-1}(t ; c) d t=0 .
\end{aligned}
$$

Thus, using the above identities, we can write

$$
\begin{aligned}
\left|\frac{(\Gamma(n / c))^{2}}{\Gamma((n / c)+r) \Gamma((n / c)-r)} G_{n, r, c}^{(\alpha, \beta)}(f ; x)-f(x)\right| \\
\leq \mid \int_{0}^{\infty}\left(\int_{x}^{t}(n-r c) \sum_{k=0}^{\infty} p_{n+r c, k}(x ; c)\right. \\
\times p_{n-(r-1) c, k+r-1}(t ; c) \\
\left.\quad \times\left[\frac{f^{\prime}\left(x^{+}\right)+f^{\prime}\left(x^{-}\right)}{2}+\left(f^{\prime}\right)_{x}(u)\right] d u\right) d t \mid \\
+\mid \int_{0}^{\infty}\left(\int_{x}^{t}(n-r c) \sum_{k=0}^{\infty} p_{n+r c, k}(x ; c)\right. \\
\times p_{n-(r-1) c, k+r-1}(t ; c) \\
\left.\times\left[\frac{f^{\prime}\left(x^{+}\right)-f^{\prime}\left(x^{-}\right)}{2} \operatorname{sgn}(u-x)\right] d u\right) d t .
\end{aligned}
$$

Also, it can be verified that

$$
\begin{aligned}
\mid \int_{0}^{\infty}\left(\int_{x}^{t}(n-r c) \sum_{k=0}^{\infty} p_{n+r c, k}(x ; c)\right. \\
\quad \times p_{n-(r-1) c, k+r-1}(t ; c) \\
\left.\quad \times\left[\frac{f^{\prime}\left(x^{+}\right)-f^{\prime}\left(x^{-}\right)}{2} \operatorname{sgn}(u-x)\right] d u\right) d t \mid \\
\leq \frac{\left|f^{\prime}\left(x^{+}\right)-f^{\prime}\left(x^{-}\right)\right|}{2}\left[U_{n, r, 2}(x)\right]^{1 / 2},
\end{aligned}
$$$$
\mid \int_{0}^{\infty}\left(\int_{x}^{t}(n-r c) \sum_{k=0}^{\infty} p_{n+r c, k}(x ; c)\right.
$$$$
\times p_{n-(r-1) c, k+r-1}(t ; c)
$$$$
\left.\times\left[\frac{f^{\prime}\left(x^{+}\right)+f^{\prime}\left(x^{-}\right)}{2}\right] d u\right) d t
$$

$$
\leq \frac{\left|f^{\prime}\left(x^{+}\right)+f^{\prime}\left(x^{-}\right)\right|}{2} U_{n, r, 1}(x) \text {. }
$$


Combining (38)-(40), we get

$$
\begin{aligned}
& \left|\frac{(\Gamma(n / c))^{2}}{\Gamma((n / c)+r) \Gamma((n / c)-r)} G_{n, r, c}^{(\alpha, \beta)}(f ; x)-f(x)\right| \\
& \leq \mid \int_{x}^{\infty}\left(\int_{x}^{t} f_{x}^{\prime}(u) d u\right)(n-r c) \\
& \quad \times \sum_{k=0}^{\infty} p_{n+r c, k}(x ; c) p_{n-(r-1) c, k+r-1}(t ; c) d t \\
& +\int_{0}^{x}\left(\int_{x}^{t} f_{x}^{\prime}(u) d u\right)(n-r c) \\
& \quad+\frac{\left|f^{\prime}\left(x^{+}\right)-f^{\prime}\left(x^{-}\right)\right|}{2}\left[U_{n, r, 2}(x)\right]^{1 / 2} \\
& \quad+\frac{\left|f^{\prime}\left(x^{+}\right)+f^{\prime}\left(x^{-}\right)\right|}{2} U_{n, r, 1}(x) \\
& \quad \times\left[U_{n, r, 2}(x)\right]^{1 / 2}+\frac{\left|f^{\prime}\left(x^{+}\right)+f^{\prime}\left(x^{-}\right)\right|}{2} U_{n, r, 1}(x) .
\end{aligned}
$$

Applying Remark 2 and Lemma 1 in above equation, we have

$$
\begin{aligned}
& \left|\frac{(\Gamma(n / c))^{2}}{\Gamma((n / c)+r) \Gamma((n / c)-r)} G_{n, r, c}^{(\alpha, \beta)}(f ; x)-f(x)\right| \\
& \leq\left|A_{n, r}^{\alpha, \beta}(f, x)\right|+\left|B_{n, r}^{\alpha, \beta}(f, x)\right| \\
& \quad+\sqrt{\frac{C \sigma_{r, c}^{\alpha, \beta}(x)}{n+\beta} \frac{\left|f^{\prime}\left(x^{+}\right)-f^{\prime}\left(x^{-}\right)\right|}{2}} \\
& \quad+\frac{\left|f^{\prime}\left(x^{+}\right)+f^{\prime}\left(x^{-}\right)\right|}{2} \\
& \quad \times\left(\frac{(\alpha-\beta x)(n-c(r+1))+2 n r c x+n x c+n r}{(n-(r+1) c)(n+\beta)}\right) .
\end{aligned}
$$

In order to complete the proof of the theorem, it suffices to estimate the terms $A_{n, r}^{\alpha, \beta}(f, x)$ and $B_{n, r}^{\alpha, \beta}(f, x)$. Applying Remark 2 with $\alpha=\beta=0$, we get

$$
\begin{aligned}
& \left|A_{n, r}^{\alpha, \beta}(f, x)\right| \\
& =\mid \int_{x}^{\infty}\left(\int_{x}^{t} f_{x}^{\prime}(u) d u\right)(n-r c) \\
& \quad \times \sum_{k=0}^{\infty} p_{n+r c, k}(x ; c) p_{n-(r-1) c, k+r-1}(t ; c) d t \mid
\end{aligned}
$$

$$
\begin{aligned}
& \leq \mid(n-r c) \sum_{k=0}^{\infty} p_{n+r c, k}(x ; c) \\
& \times \int_{2 x}^{\infty}(f(t)-f(x)) p_{n-(r-1) c, k+r-1}(t ; c) d t|+| f^{\prime}\left(x^{+}\right) \mid \\
& \times \mid(n-r c) \sum_{k=0}^{\infty} p_{n+r c, k}(x ; c) \\
& \times \int_{x}^{2 x} p_{n-(r-1) c, k+r-1}(t ; c)(t-x) d t \mid \\
& +\left|\int_{x}^{2 x} f_{x}^{\prime}(u) d u\right|\left|1-\lambda_{n, r}(x, 2 x)\right| \\
& +\left|\int_{x}^{2 x}\right| f_{x}^{\prime}(t)|\cdot| 1-\lambda_{n, r}(x, t)|d t| \\
& \leq(n-r c) \sum_{k=0}^{\infty} p_{n+r c, k}(x ; c) \\
& \times \int_{2 x}^{\infty} p_{n-(r-1) c, k+r-1}(t ; c) C_{1} t^{2 q} d t \\
& +\frac{|f(x)|}{x^{2}}(n-r c) \sum_{k=0}^{\infty} p_{n+r c, k}(x ; c) \\
& \times \int_{0}^{\infty} p_{n-(r-1) c, k+r-1}(t ; c)(t-x)^{2} d t \\
& +\left|f^{\prime}\left(x^{+}\right)\right|(n-r c) \sum_{k=0}^{\infty} p_{n+r c, k}(x ; c) \\
& \times \int_{2 x}^{\infty} p_{n-(r-1) c, k+r-1}(t ; c)|t-x| d t \\
& +\frac{C x(1+c x)}{n x^{2}}\left|f(2 x)-(x)-x f^{\prime}\left(x^{+}\right)\right| \\
& +\frac{C(1+c x)}{n} \sum_{k=1}^{[\sqrt{n}]} \bigvee_{x}^{x+(x / k)}\left(f_{x}^{\prime}(x)\right) \\
& +\frac{x}{\sqrt{n}} \bigvee_{x}^{x+(x / \sqrt{n})}\left(f_{x}^{\prime}(x)\right)
\end{aligned}
$$

For estimating the integral

$$
(n-r c) \sum_{k=0}^{\infty} p_{n+r c, k}(x ; c) \int_{2 x}^{\infty} p_{n-(r-1) c, k+r-1}(t ; c) C_{1} t^{2 q} d t
$$


we proceed as follows: since $t \geq 2 x$ implies that $t \leq 2(t-x)$ so by Schwarz inequality and Lemma 1,

$$
\begin{aligned}
(n-r c) & \sum_{k=0}^{\infty} p_{n+r c, k}(x ; c) \int_{2 x}^{\infty} p_{n-(r-1) c, k+r-1}(t ; c) C_{1} t^{2 q} d t \\
\leq & C_{1} 2^{q}(n-r c) \sum_{k=0}^{\infty} p_{n+r c, k}(x ; c) \\
& \times \int_{0}^{\infty} p_{n-(r-1) c, k+r-1}(t ; c) C_{1}(t-x)^{2 q} d t \\
\leq & C_{1} 2^{q} U_{n, r, 2 q}(x)=O\left(n^{-q}\right) \quad \text { as } n \longrightarrow \infty .
\end{aligned}
$$

By using Hölder's inequality and Remark $2(\alpha=\beta=0)$, we get the estimate as follows:

$$
\begin{aligned}
& \left|f^{\prime}\left(x^{+}\right)\right|(n-r c) \sum_{k=0}^{\infty} p_{n+r c, k}(x ; c) \\
& \quad \times \int_{2 x}^{\infty} p_{n-(r-1) c, k+r-1}(t ; c)|t-x| d t \\
& \leq\left|f^{\prime}\left(x^{+}\right)\right| \\
& \quad \times\left((n-r c) \sum_{k=0}^{\infty} p_{n+r c, k}(x ; c)\right. \\
& \left.\quad \times \int_{0}^{\infty} p_{n-(r-1) c, k+r-1}(t ; c)(t-x)^{2} d t\right)^{1 / 2} \\
& \leq\left|f^{\prime}\left(x^{+}\right)\right| \sqrt{\frac{C x(1+c x)}{n}} .
\end{aligned}
$$

Collecting the estimates from (43)-(46), we obtain

$$
\begin{aligned}
\left|A_{n, r}^{\alpha, \beta}(f, x)\right| \leq & O\left(n^{-q}\right)+\left|f^{\prime}\left(x^{+}\right)\right| \\
& \times \sqrt{\frac{C x(1+c x)}{n}}+\frac{C(1+c x)}{n x} \\
& \times\left|f(2 x)-f(x)-x f^{\prime}\left(x^{+}\right)+\right| f(x) \mid \\
& +\frac{C(1+c x)}{n} \sum_{k=1}^{[\sqrt{n}]} \bigvee_{x}^{x+(x / k)}\left(f_{x}^{\prime}(x)\right) \\
& +\frac{x}{\sqrt{n}} \bigvee_{x}^{x+(x / \sqrt{n})}\left(f_{x}^{\prime}(x)\right) .
\end{aligned}
$$

On the other hand, to estimate $B_{n, r}^{\alpha, \beta}(f, x)$ by applying Lemma 4 with $y=x-(x / \sqrt{n})$ and integration by parts, we have

$$
\begin{aligned}
\left|B_{n, r}^{\alpha, \beta}(f, x)\right| & \left|\int_{0}^{x} \int_{x}^{t} f_{x}^{\prime}(u) d_{t} \lambda_{n, r}(x, t)\right| \\
\leq & \left(\int_{0}^{y}+\int_{y}^{x}\right)\left|f_{x}^{\prime}(t)\right|\left|\lambda_{n, r}(x, t)\right| d t \\
\leq & \frac{C x(1+c x)}{n} \int_{0}^{y} \bigvee_{t}^{x}\left(\left(f^{\prime}\right)_{x}\right) \frac{1}{(x-t)^{2}} d t \\
& +\int_{y}^{x} \bigvee_{t}^{x}\left(\left(f^{\prime}\right)_{x}\right) d t \\
= & \frac{C x(1+c x)}{n} \int_{1}^{\sqrt{n}} \bigvee_{(x-(x / u))}^{x}\left(\left(f^{\prime}\right)_{x}\right) d u \\
& +\frac{x}{\sqrt{n}} \bigvee_{x-(x / \sqrt{n})}^{x}\left(\left(f^{\prime}\right)_{x}\right) \\
\leq & \frac{C x(1+c x)}{n} \sum_{k=1}^{[\sqrt{n}]} \bigvee_{x-(x / k)}^{x}\left(\left(f^{\prime}\right)_{x}\right) \\
& +\frac{x}{\sqrt{n}} \bigvee_{x-(x / \sqrt{n})}^{x}\left(\left(f^{\prime}\right)_{x}\right),
\end{aligned}
$$

where $u=(x /(x-t))$.

Combining (41), (47), and (48), we get the desired result.

Corollary 7. Let $f^{(s)} \in D B_{q}(0, \infty), q>0$, and $x \in(0, \infty)$. Then, for $C>2$ and $n$ sufficiently large, one has

$$
\begin{aligned}
& \mid \frac{(\Gamma(n / c))^{2}}{\Gamma((n / c)+r) \Gamma((n / c)-r)}\left(\frac{n+\beta}{n}\right)^{s} \\
& \times D^{s} G_{n, r, c}^{(\alpha, \beta)}(f ; x)-f^{s}(x) \mid \\
& \leq \frac{C(1+c x)}{n} \sum_{k=1}^{[\sqrt{n}]} \bigvee_{x-(x / k)}^{x+(x / k)}\left(\left(D^{s+1} f\right)_{x}\right) \\
& +\frac{x}{\sqrt{n}} \bigvee_{x-(x / \sqrt{n})}^{x+(x / \sqrt{n})}\left(\left(D^{s+1} f\right)_{x}\right)+\frac{C(1+c x)}{n x} \\
& \quad \times\left|f(2 x)-(x)-x D^{s+1} f\left(x^{+}\right)+\right| f(x)|| \\
& +O\left(n^{-q}\right)+\left|D^{s+1} f\left(x^{+}\right)\right| \sqrt{\frac{C x(1+c x)}{n}}
\end{aligned}
$$




$$
\begin{aligned}
& +\sqrt{\frac{C \sigma_{r, c}^{\alpha, \beta}(x)}{n+\beta} \frac{\left|D^{s+1} f\left(x^{+}\right)-D^{s+1} f\left(x^{-}\right)\right|}{2}} \\
& +\frac{\left|D^{s+1} f\left(x^{+}\right)+D^{s+1} f\left(x^{-}\right)\right|}{2} \\
& \times\left(\frac{(\alpha-\beta x)(n-c(r+1))+2 n r c x+n x c+n r}{(n-(r+1) c)(n+\beta)}\right),
\end{aligned}
$$

where $\bigvee_{a}^{b} f_{x}$ denotes the total variation of $f_{x}$ on $[a, b]$ and the auxiliary function $D^{s+1} f_{x}$ is defined by

$$
D^{s+1} f_{x}(t)= \begin{cases}D^{s+1} f(t)-D^{s+1} f\left(x^{-}\right), & 0 \leq t \leq x \\ 0, & t=x \\ D^{s+1} f(t)-D^{s+1} f\left(x^{+}\right), & x<t<\infty .\end{cases}
$$

\section{Conclusion}

The results of our lemmas and theorems are more general rather than the results of any other previously proved lemmas and theorems, which will enrich the literature of applications of quantum calculus in operator theory and convergence estimates in the theory of approximations by positive linear operators. The researchers and professionals working or intend to work in areas of mathematical analysis and its applications will find this research paper to be quite useful. Consequently, the results so established may be found useful in several interesting situations appearing in the literature on mathematical analysis, pure and applied mathematics, and mathematical physics. Some interesting applications of the positive approximation linear operators can be seen in [2124].

\section{Conflict of Interests}

The authors declare that there is no conflict of interests regarding the publication of this paper.

\section{Acknowledgments}

The authors would like to express their deep gratitude to the anonymous learned referee(s) and the editor for their valuable suggestions and constructive comments, which resulted in the subsequent improvement of this research paper. Special thanks are due to Professor Józef Banaś, Editor of the Journal of Function Spaces, for his efforts to send the reports of the paper timely. The authors are also grateful to all the editorial board members and reviewers of esteemed journal, that is, Journal of Function Spaces. The second author Lakshmi Narayan Mishra acknowledges the Ministry of Human Resource Development (MHRD), New Delhi, India, for supporting this research paper at the Department of Mathematics, National Institute of Technology (NIT), Silchar, Assam. The third author Vishnu Narayan Mishra acknowledges that this paper project was supported by Sardar
Vallabhbhai National Institute of Technology (SVNIT), Surat (Gujarat), India. All the authors carried out the proof of theorems. Each author contributed equally in the development of the paper. Vishnu Narayan Mishra conceived of the study and participated in its design and coordination. All the authors read and approved the final version of paper.

\section{References}

[1] H. M. Srivastava and V. Gupta, "A certain family of summationintegral type operators," Mathematical and Computer Modelling, vol. 37, no. 12-13, pp. 1307-1315, 2003.

[2] N. Ispir and I. Yuksel, "On the Bezier variant of SrivastavaGupta operators," Applied Mathematics E-Notes, vol. 5, pp. 129137,2005

[3] V. Gupta and M. Ivan, "Rate of simultaneous approximation for the Bézier variant of certain operators," Applied Mathematics and Computation, vol. 199, no. 1, pp. 392-395, 2008.

[4] N. Deo, "Faster rate of convergence on Srivastava-Gupta operators," Applied Mathematics and Computation, vol. 218, no. 21, pp. 10486-10491, 2012.

[5] D. K. Verma and P. N. Agrawal, "Convergence in simultaneous approximation for Srivastava-Gupta operators," Mathematical Sciences, vol. 6, article 22, 2012.

[6] T. Acar, "Rate of convergence for generalized Szasz operators with derivatives of bounded variation," Proceedings of the Jangjeon Mathematical Society, vol. 16, no. 1, pp. 21-35, 2013.

[7] T. Acar, V. Gupta, and A. Aral, "Rate of convergence for generalized Szász operators," Bulletin of Mathematical Sciences, vol. 1, no. 1, pp. 99-113, 2011.

[8] N. İspir, A. Aral, and O. Doğru, "On Kantorovich process of a sequence of the generalized linear positive operators," Numerical Functional Analysis and Optimization, vol. 29, no. 5-6, pp. 574-589, 2008.

[9] V. Gupta, "Rate of approximation by a new sequence of linear positive operators," Computers \& Mathematics with Applications, vol. 45, no. 12, pp. 1895-1904, 2003.

[10] V. Gupta, N. Deo, and X. Zeng, "Simultaneous approximation for Szasz-Mirakian-STAncu-Durrmeyer operators," Analysis in Theory and Applications, vol. 29, no. 1, pp. 86-96, 2013.

[11] D. D. Stancu, "Approximation of functions by a new class of linear polynomial operators," Revue Roumaine de Mathématique Pures et Appliquées, vol. 13, pp. 1173-1194, 1968.

[12] D. D. Stancu, "Approximation of functions by means of a new generalized Bernstein operator," Calcolo, vol. 20, no. 2, pp. 211229, 1983.

[13] V. N. Mishra, K. Khatri, L. N. Mishra, and Deepmala, "Inverse result in simultaneous approximation by Baskakov-DurrmeyerStancu operators," Journal of Inequalities and Applications, vol. 2013, article 586, 2013.

[14] V. N. Mishra, H. H. Khan, K. Khatri, and L. N. Mishra, "Hypergeometric representation for Baskakov-Durrmeyer-Stancu type operators," Bulletin of Mathematical Analysis and Applications, vol. 5, no. 3, pp. 18-26, 2013.

[15] V. N. Mishra, K. Khatri, and L. N. Mishra, "On simultaneous approximation for Baskakov-Durrmeyer-Stancu type operators," Journal of Ultra Scientist of Physical Sciences, vol. 24, no. 3, pp. 567-577, 2012.

[16] V. N. Mishra, K. Khatri, and L. N. Mishra, "Statistical approximation by Kantorovich-type discrete q-beta operators," Advances in Difference Equations, vol. 2013, article 345, 2013. 
[17] V. N. Mishra, K. Khatri, and L. N. Mishra, "Some approximation properties of $q$-Baskakov-Beta-Stancu type operators," Journal of Calculus of Variations, vol. 2013, Article ID 814824, 8 pages, 2013.

[18] V. N. Mishra, K. Khatri, L. N. Mishra, and Deepmala, "Trigonometric approximation of periodic Signals belonging to generalized weighted Lipschitz $W\left(L_{r}, \xi(t)\right),(r \geq 1)$-class by NörlundEuler $\left(N, p_{n}\right)(E, q)$ operator of conjugate series of its Fourier series, accepted for publication in Journal of Classical Analysis," Journal of Classical Analysis. In press.

[19] L. N. Mishra, V. N. Mishra, K. Khatri, and Deepmala, "On the trigonometric approximation of signals belonging to generalized weighted Lipschitz $W\left(L^{r}, \xi(t)\right)(r \geq 1)$-class by matrix $\left(C^{1}\right.$. $N_{p}$ ) operator of conjugate series of its Fourier series," Applied Mathematics and Computation, vol. 237, pp. 252-263, 2014.

[20] V. N. Mishra and L. N. Mishra, "Trigonometric approximation in $L_{p}(p \geq 1)$-spaces," International Journal of Contemporary Mathematical Sciences, vol. 7, no. 12, pp. 909-918, 2012.

[21] Deepmala and H. K. Pathak, "A study on some problems on existence of solutions for nonlinear functional-integral equations," Acta Mathematica Scientia, vol. 33, no. 5, pp. 13051313, 2013.

[22] Deepmala, A study on fixed point theorems for nonlinear contractions and its applications [Ph.D. thesis], Pt. Ravishankar Shukla University, Raipur, India, 2014.

[23] L. N. Mishra, S. K. Tiwari, V. N. Mishra, and V. Mishra, "Unique fixed point theorems for generalized contractive mappings in partial metric spaces," Journal of Function Spaces, vol. 2014, Article ID 960827, 15 pages, 2014.

[24] Deepmala, "Existence theorems for solvability of a functional equation arising in dynamic programming," International Journal of Mathematics and Mathematical Sciences, vol. 2014, Article ID 706585, 9 pages, 2014. 


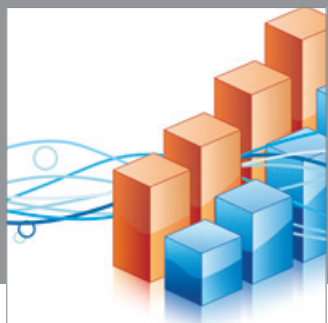

Advances in

Operations Research

mansans

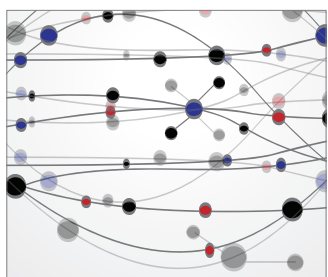

The Scientific World Journal
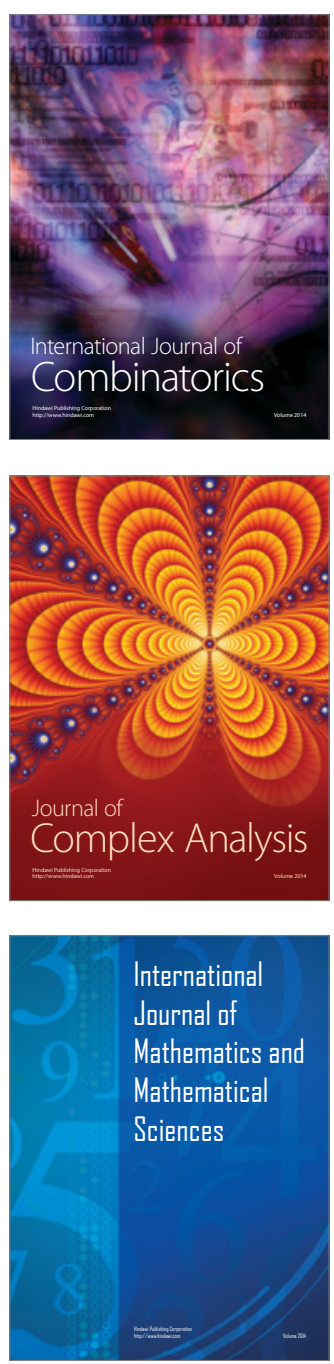
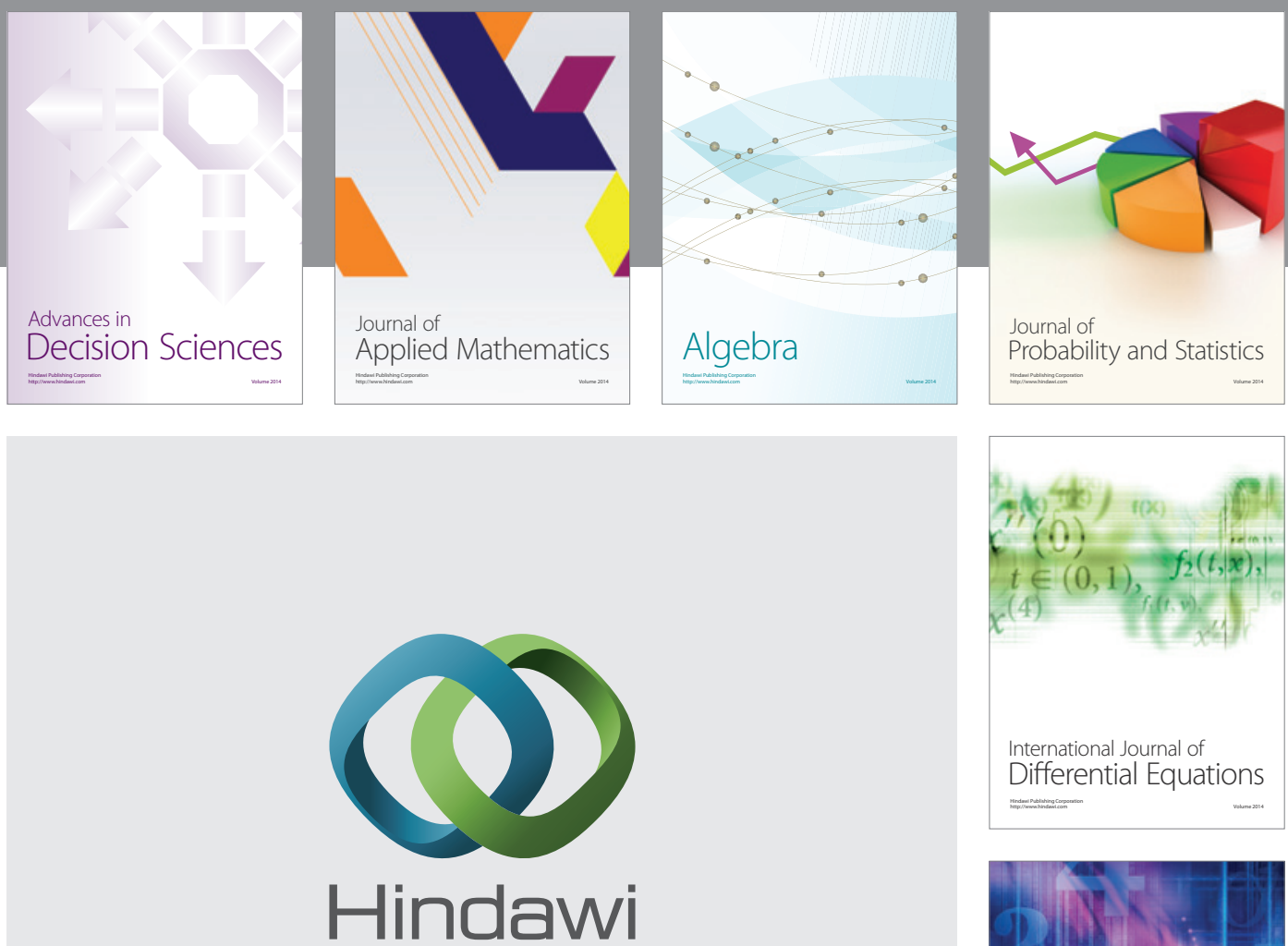

Submit your manuscripts at http://www.hindawi.com
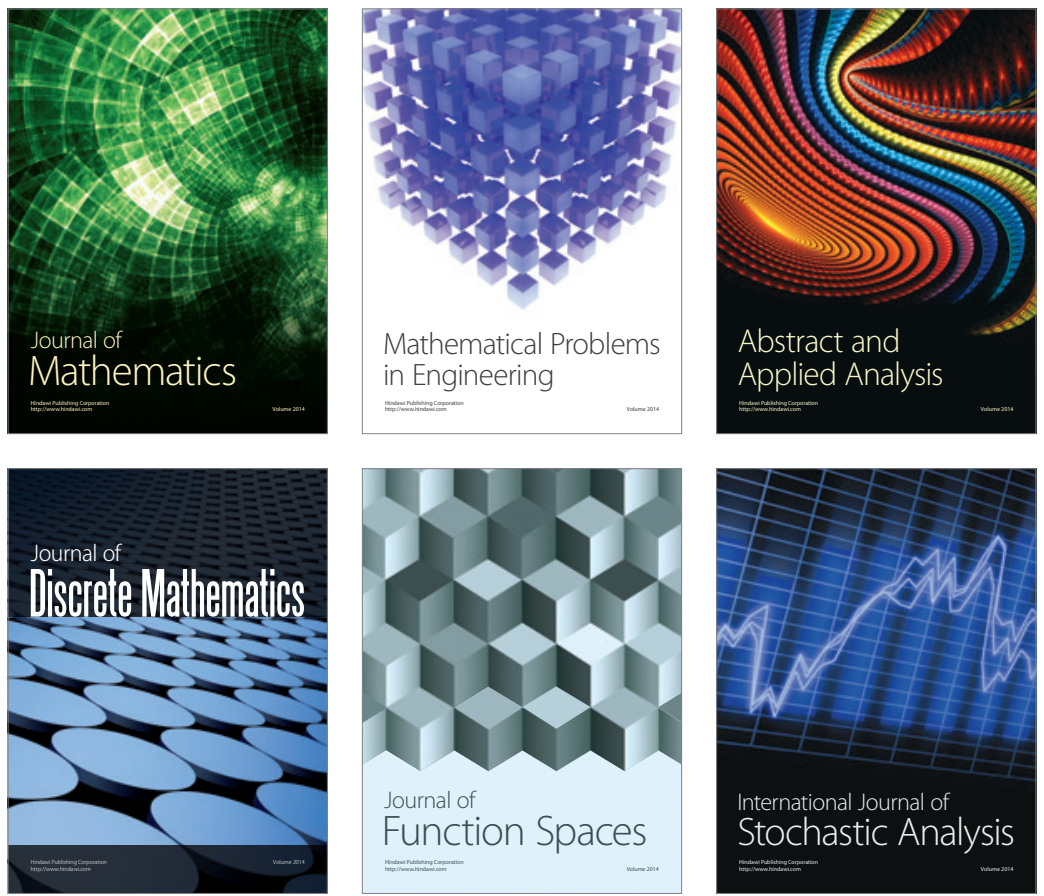

Journal of

Function Spaces

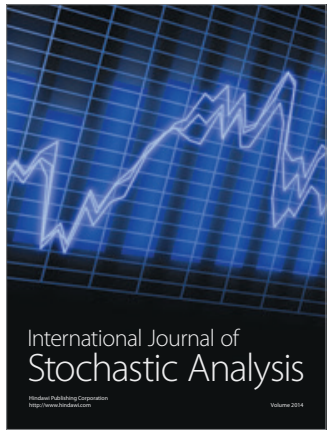

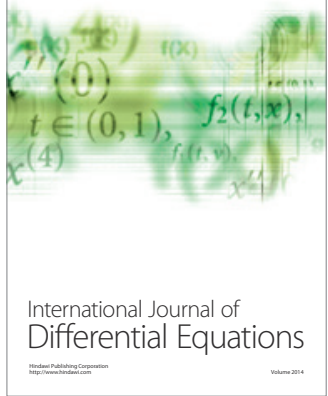
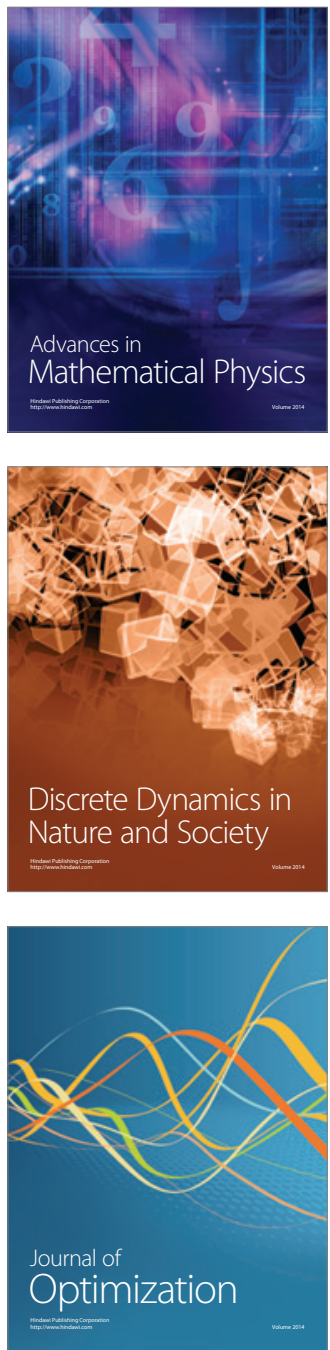